\title{
A GRASP-based Heuristic for Allocating the Roadside Infrastructure Maximizing the Number of Distinct Vehicles Experiencing Contact Opportunities
}

\author{
João F. Machry Sarubbi \\ CEFETMG \\ Departamento de Computação \\ Belo Horizonte, MG, Brazil \\ joao@decom.cefetmg.br
}

\author{
Daniel Cravieé de A. Vieira \\ CEFETMG \\ Departamento de Computação \\ Belo Horizonte, MG, Brazil \\ danielcraviee@gmail.com
}

\author{
Elizabeth F. Wanner \\ CEFETMG
}

Departamento de Computação
Belo Horizonte, MG, Brazil

efwanner@decom.cefetmg.br
Cristiano M. Silva

UFSJ

\begin{abstract}
In this work the allocation of Roadside Units (RSUs) in a V2I network is modeled as a Maximum Coverage Problem. The main objective is to maximize the number of distinct vehicles contacting the infrastructure. Two different approaches are presented to solve the problem. The first one is an ILP model that can found optimal solutions or give sharp upper and lower bounds for the problem. The second one is a GRASP-based heuristic that can found close-to-optimal solutions. The GRASP-based heuristic is compared with a previous work achieving better results. Furthermore, a new metric to measure the efficiency of a Deployment strategy is presented.
\end{abstract}

Index Terms-Vehicular Networks; Deployment Strategies; GRASP Heuristic; ILP model;

\section{INTRODUCTION}

Vehicular Networks (VANETS) [1] [2] are wireless communication networks that support cooperative driving among communicating vehicles on the road. According to Karagiannis et al. [3] "vehicular connectivity techniques can significantly enhance efficiency of travel, reduce traffic incidents and improve safety, mitigate the impact of congestion". The VANETS involve vehicle-to-vehicle (V2V) and vehicle-to-infrastructure (V2I) communications. In a V2V communication, the vehicles exchange messages without any support infrastructure [4]. However, the V2V communication may become inefficient in sparse areas such as highways, rural zones and low peak hours in the city due to the lack of communicating pairs and radio obstacles. On the other hand, several research studies demonstrate that a minimum support infrastructure, i.e., the usage of a V2I network, may largely improve the overall efficiency of the vehicular network [5]-[8].

Despite the advantages of an infrastructure vehicular network, the main drawback of a V2I network is the need of installing the Roadside Units (RSUs), which are usually expensive $[8,9]$, turning the decision related to the amount and location of RSUs a challenge to planning authorities. On the other hand, these planning authorities want that a maximum among of vehicles be connected to some RSU during its trip. 978-1-5090-0223-8/16/\$31.00 (c) 2016 IEEE
However, due to the RSU costs, these authorities usually have a restrict number of available RSUs.

In this work a problem to deploy RSUs is tackled in order to maximize the number of distinct vehicles contacting the infrastructure, an interesting metric when we intend to collect and disseminate small and self-contained traffic announcements [10]. It is assumed the vehicle will receive the information if it gets in contact with a RSU at least once. Under this assumption, the goal is to place the RSUs at $\eta$ of the possible intersections so as to maximize the number of vehicles that enter a RSU coverage area at least once.

In this work two different approaches to solve the problem are proposed. Both of them consider the density and the mobility information of vehicles along the urban area. The first approach is an Integer Linear Programming (ILP) model that can compute exact solutions and/or give lower and upper bounds to the problem. The second one is a GRASP-like heuristic. Both approaches are compared with the MCP-g algorithm proposed by Trullols et al. [10]. A new metric to measure the efficiency of a Deployment strategy is also proposed. Results demonstrate that the use of more sophisticated techniques, such as GRASP based heuristics, can get better results than just simple heuristics even when these simple heuristics already found close-to-optimal solutions.

This work is organized as follows: Section II presents a selection of related work. Section III presents the novel proposal to represent complex road network. Section IV formalizes the Deployment as a Maximum Coverage Problem. Section V presents the baseline algorithm. Section VI presents the proposed solution. Section VII presents the experiments. Section VIII concludes the work.

\section{RELATED WORK}

Researchers have been studying the allocation of roadside units in vehicular networks through several points of view. The characterization of the vehicular mobility is certainly a key challenge. Yong Li et al. [11] examine the predictability 
limits of large-scale urban vehicular networks by using the entropy theory, while [12] investigates the spatial distribution of taxis in Shanghai. Authors discuss how very popular roads impact the vehicle-to-infrastructure contact time distribution.

Strategies for content download between roadside units and vehicles are also analyzed in literature: Sadiq et al. [13] propose an intelligent network selection strategy based on the Faded Signal-to-Noise Ratio, Residual Channel Capacity, and Connection Life Time with the goal of reducing the delays for streaming applications. Cruces et al. [14] introduce a mixed-integer quadratic programming based optimum roadside units deployment scheme to provide Internet access services for the maximum road traffic volumes with limited number of roadside units. Liu et al. [15] propose a new roadside units deployment strategy for file download.

The use of existing network infrastructures is also investigated: Marfia et al. [16] propose the use of open Access Points. Tonguz and Viriyasitavat [17] propose the utilization of vehicles as roadside units by using a biologically inspired network. Liang and Zhuang [18] propose the use of the wireless LAN for data dissemination.

There are also works proposing deployment strategies: Lee and Kim [19] propose a greedy heuristic to place the roadside units aiming to improve vehicles connectivity while reducing disconnections. The heuristic counts the amount of reached vehicles at each intersection considering the transmission range of the roadside units. Jeonghee et al. [20] propose a deployment based on the intersection connectivity. Barrachina et al. [9] present three RSUs deployment policies: (i) the Minimum Cost which considers only the cost to install the RSUs. This strategy priorizes locations that already have Internet access leaving that some areas remain isolated; (ii) the Uniform Mesh which consist on distributing RSUs uniformly on the map. This strategy reduces the probability of having shadow areas in the map but not taking into account the real flow of vehicles that traveling around the city; (iii) the D-RSU deployment in which "RSUs are placed using an inverse proportion to the expected density". The authors consider that vehicles can use Vehicle to Vehicle (V2V) communication and that the RSUs are more important in low density areas. Barrachina et al. [2] present an architecture to estimate traffic density that combines V2V and V2I communication. They use a roadmap topology features from real cities and uses a ns-simulator to estimate the traffic. Kchiche and Kamoun [8] use a centrality and equidistant-based (uniform) deployment to optimize the delay and ensure a regular and stable service in a V2I and V2V network. Trullols et al. [10] formulate the allocation of roadside units as a Maximum Coverage Problem. The objective was to maximize the number of distinct vehicles experiencing (at least one) V2I contact opportunities. They present a greedy heuristic named MCP-g which was compared with other heuristics but was not compared with the optimal solution. Silva et al. [21] also aim to maximize the number of distinct vehicles experiencing (at least one) V2I contact opportunities. They present a deployment strategy that, different from Trullols et al. [10], does not uses the full knowledge of the vehicle trajectories to achieve a close-to-optimal deployment performance.

\section{REPRESENTING ROAD NETWORKS}

Before applying the deployment strategies, it is necessary to represent road networks of arbitrary topology. In this study the urban area is partitioned into a set of adjacent cells of same size (i.e., grid model). Once the region is partitioned, the original road network is discarded and the flow between adjacent grid cells is manipulated. Partition is a strategy which represents a road network and its associated flow by a grid structure of arbitrary granularity. When more/less accuracy is needed, it is only necessary to increase/decrease the number of grid cells inside the region.

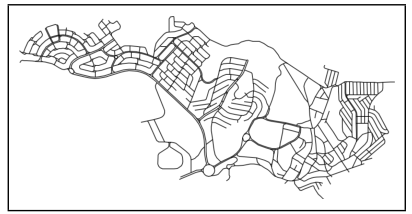

(a) Road Network.

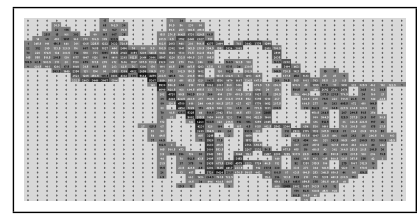

(c) $40 \times 40$ grid.

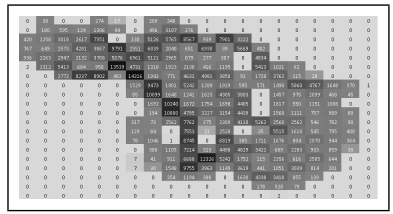

(b) $20 \times 20$ grid

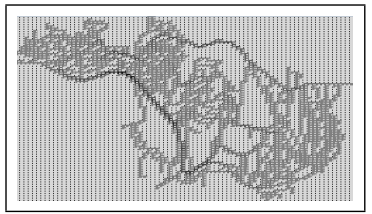

(d) $80 \times 80$ grid.
Figure 1. Distinct Grid Setups.

Figure 1(a) shows a real road network (Ouro Branco city, Brazil). Figures 1(b) to 1(d) show how such road network may be modeled by grid setups from $20 \times 20$ up to $80 \times 80$. Inside each urban cell, the number of vehicles crossing it during a given time interval is indicated. The resolution may be changed arbitrarily.

\section{Problem Definition}

Given a set of vehicles $K, K=\{1,2, \ldots, k\}$, a set of road network partitions $C, C=\{1,2, . ., c\}$, the full vehicles trajectories information $G$ and the number $\eta$ of available roadside units, the goal is to choose the best set of urban cells to install the RSUs in order to maximize the number of distinct vehicles contacting the RSUs. Each vehicle can contact more than one RSU and each RSU can contact more than one vehicle. However, as the goal is to maximize the distinct vehicles, if more than one RSU contacts the same vehicle, this vehicle will be counted just one time.

As Trullols et al. [10], the deployment of roadside units is also modeled as a Maximum Coverage Problem.

Definition 1 (Maximum Coverage Problem). Suppose a collection of sets $S=\left\{S_{1}, S_{2}, \ldots, S_{m}\right\}$ defined over a domain of elements $X=\left\{x_{1}, x_{2}, \ldots, x_{n}\right\}$. Sets may share elements. The goal is to find a collection of sets $S^{\prime} \subseteq S$ such that the number of covered elements $\left|\bigcup_{S_{i} \in S^{\prime}}\right|$ is maximized. 
For that Deployment, the sets $S$ are the RSUs and the elements $X$ are the vehicles. For this problem, an Integer Linear Programming (ILP) formulation $M$ with the following sets of variables is defined:

$$
\begin{aligned}
& a_{c}= \begin{cases}1, & \text { if urban cell } c \text { receives a roadside unit } \\
0, & \text { otherwise. }\end{cases} \\
& v_{k}= \begin{cases}1, & \text { if vehicle } k \text { has crossed a roadside unit } \\
0, & \text { otherwise. }\end{cases}
\end{aligned}
$$

and the following set of parameters:

$$
m_{c k}= \begin{cases}1, & \text { if vehicle } k \text { crosses urban cell } c \\ 0, & \text { otherwise }\end{cases}
$$

$\eta$ : number of install RSUs.

The deployment of roadside units is modeled as follows:

$$
\max \sum_{n=1}^{k} v_{n}
$$

Subject to:

$$
\begin{array}{cc}
\sum_{\forall c \in C, \forall k \in K \mid} a_{c} \leq \eta & \\
\sum_{M_{c k}=1} a_{c} \geq v_{k} & \forall k \in K \\
v_{k} \geq A_{c k} \quad \forall c \in C, \forall k \in K \mid m_{c k}=1 \\
a_{c} \in\{0,1\} \\
a_{k} \in\{0,1\}
\end{array}
$$

Objective function (1) maximizes the number of distinct vehicles reaching roadside units. Constraint (2) ensures that number of selected urban cells is $\leq \eta$. Constraint (3) ensures that whenever the vehicle $k$ is covered, at least one of the urban cell crossed by $k$ has a roadside unit. Constraint (4) ensures that whenever the vehicle $k$ crosses an urban cell having a roadside unit, then the vehicle $k$ is covered. Constraints (5) and (6) are the integrality constraints.

\section{BAseline Algorithm}

In this section the baseline algorithm proposed by Trullols et al. [10] is presented. Although Maximum Coverage Problem is NP-Hard, it is well-known that the greedy heuristic achieves an approximation factor of $(1-1 / m)^{m}$, in which $m$ is the maximum cardinality of the sets in the optimization domain [22]. Trullols et al. [10] named this heuristic as MCP-g. In order to cover a given region, MCP-g iteratively selects those $\eta$ urban cells having the largest number of uncovered vehicles.

MCP-g receives as input the full trajectories information $G$ and the number of available roadside units $\eta$. It chooses sets (i.e., urban cells) according to one rule: at each stage, choose a set which contains the largest number of uncovered elements. Trullols et al. present an in-depth discussion of MCP-g in [10].

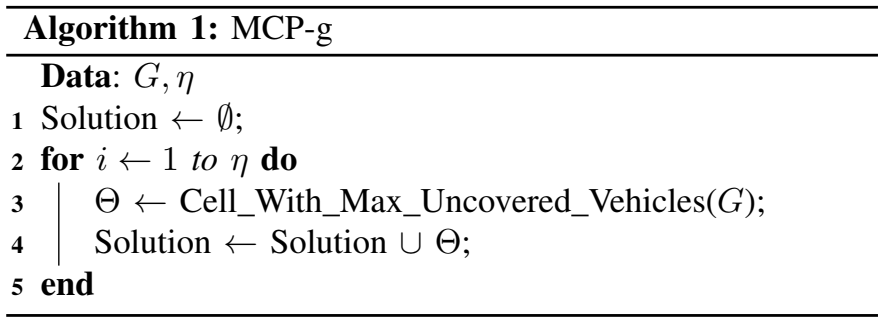

\section{MCP_GRASP ALGORITHM}

In this section the proposed strategy to solve the problem is presented. A version of the well known Greedy Randomized Adaptive Search Procedure (GRASP) [23], named MCP_GRASP algorithm, is used. This metaheuristic consists of two phases: (a) Construction Phase; (b) Local Search Phase. Both phases are repeated for each iteration. Construction Phase consists of a randomized greedy function building up an initial solution. Solution is then used in the Local Search. Final result is simply the best solution found over all iterations. Algorithm 2 presents the MCP_GRASP algorithm.
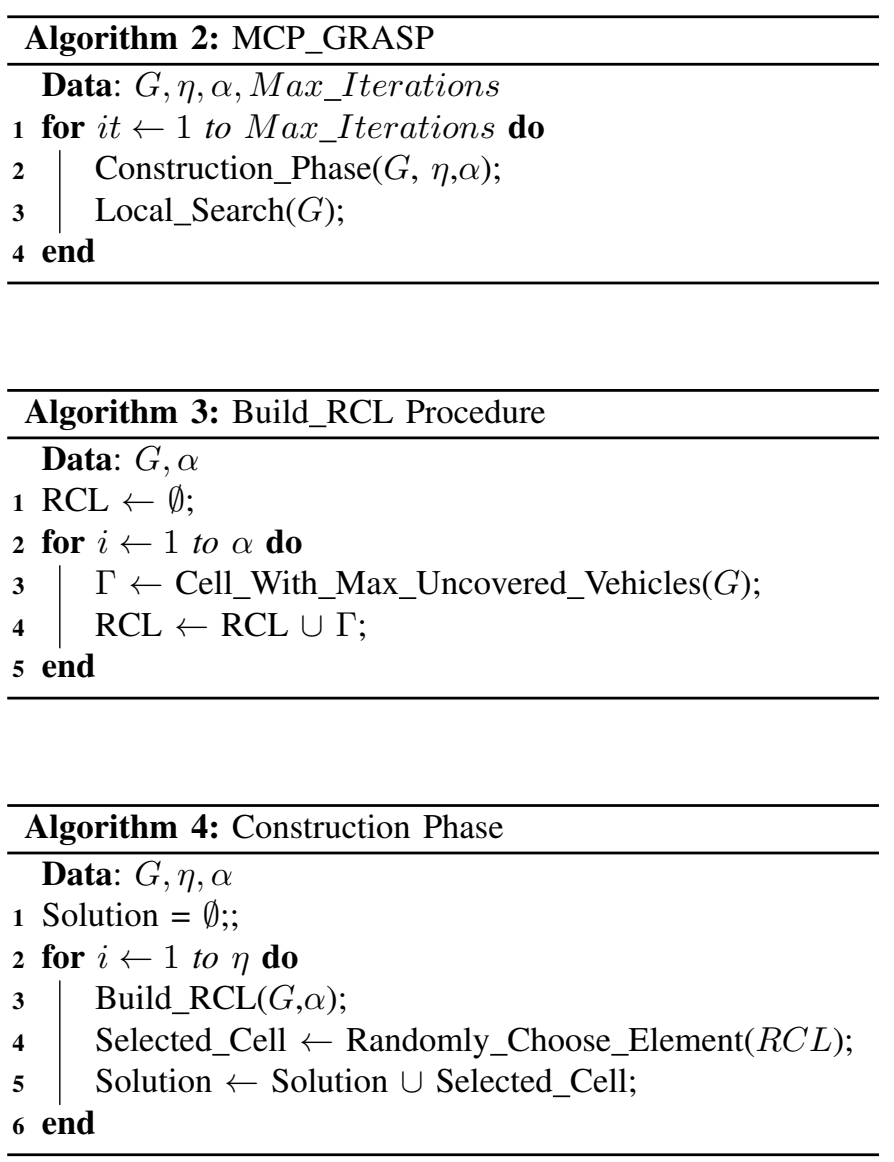

In Construction Phase, a randomized greedy technique provides feasible solutions. This feasible solution is iteratively constructed, one element at a time. For the Construction Phase it is implemented a variation of the MCP-g algorithm 
proposed by [10]. In the proposed algorithm, instead of always selecting the best solution (the cell with max uncovered vehicles), a Restricted Candidate List (RCL) of good elements is built, and one element (not necessarily the top candidate) is ramdomly selected. A RCL parameter $\alpha$ determines the level of greediness or randomness in the Construction Phase. In the algorithm, $\alpha$ is the RCL size. When $\alpha=1$, the $\mathrm{RCL}$ has one element representing a full greedy solution, i.e., the Construction Phase becomes the MCP-g algorithm. When $\alpha=2$, the RCL has two elements, that means that the RCL will have the two urban cell with max uncovered vehicles, and so forth. For instance, when $\alpha=N$, a full random solution is returned. Algorithms 3 and 4 present the proposed Build_RCL and Construction Phase algorithms.

Algorithm 5 presents the Local Search Phase. After receiving the $\eta$ RSUs chosen by the Construction Phase, successive exchanges are performed. For each RSU, all possible neighborhood urban cells are tested. If with one exchange a better solution (more achieved vehicles) is achieved, the Local Search Phase is restarted with the new set of RSUs. The neighborhood urban cells are presented in Figure 2.
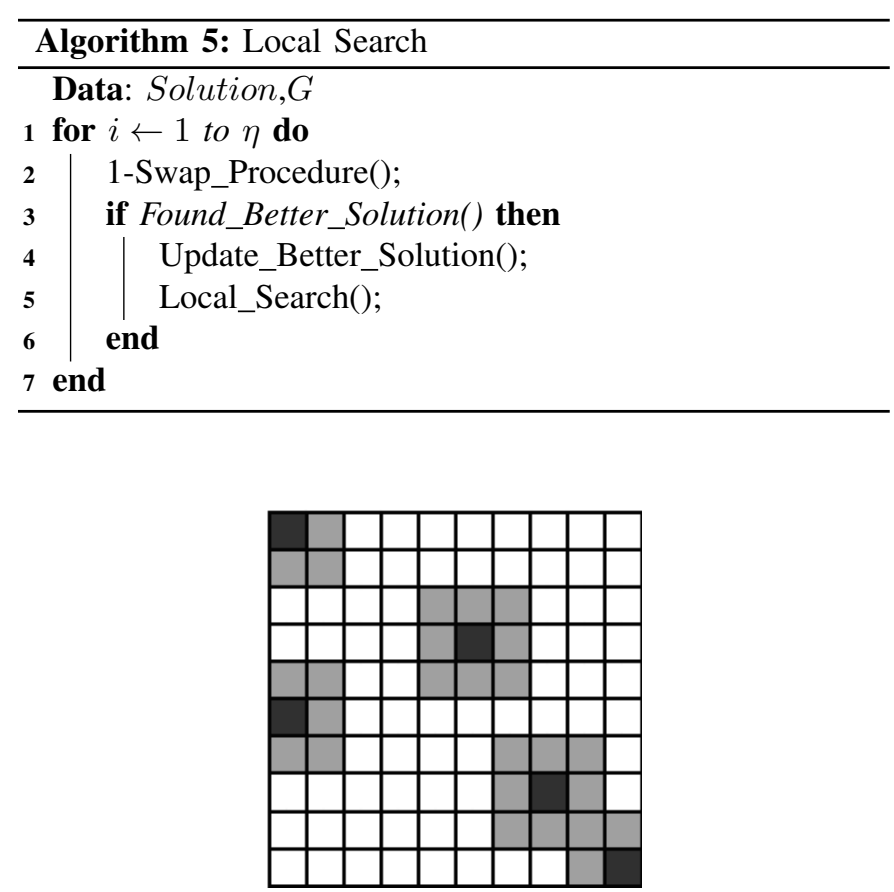

Figure 2. Local Search Neighborhood

\section{EXPERIMENTS}

In this Section, the experiments comparing the results of the Baseline algorithm MCP-g with the proposed MCP_GRASP algorithm and Integer Linear Model using solver CPLEX is shown. Experiments are based on the realistic mobility trace (http://kolntrace.project.citi-lab.fr/) of the Cologne, Germany. The trace is composed of 10,000s of traffic from 75515 vehicles. All experiments are performed using the SUMO simulator http://sumo-sim.org) and a set of tools designed by our team. SUMO runs the Cologne scenario and outputs the location of each vehicle (our mobility trace $T$ ) over time. The Partition Program reads the mobility trace, computes the bounding box of the mobility trace, partitions the Cologne into a grid of $\psi \times \psi$ urban cells, and then translates the mobility trace from Cartesian coordinates to Grid coordinates. For all experiments, $\psi=100$ is used.

Programs MCP-g, MCP_GRASP and CPLEX are run with different values for $\eta$ : each of these programs outputs an individual file containing the position of the roadside units according to each strategy. Then, the Analysis Program is run: it receives as input the mobility trace and a file containing the location of the roadside units, and outputs several reports used to evaluate our deployment performance.

For each $\eta$ value, the MCP_GRASP algorithm is run 5 times. Each time a different value for $\alpha$ is used. The $\alpha$ value varies from 1 to 5 . At each time, Max_Iterations is set to 100. The MCP_GRASP solution is simply the best solution found over all tested $\alpha$ values. CPLEX is used with default parameters and CPLEX upper bound is initiated with the best solution found by MCP_GRASP algorithm. A limit of $8 \mathrm{hs}$ is imposed to CPLEX compute the solution.

\section{A. Comparing CPLEX, MCP-g and MCP_GRASP algorithms}

The first set of experiments compares the three different approaches to solve the problem, the CPLEX solver, the MCP-g algorithm and the MCP_GRASP implementation. All approaches are tested for different $\eta$ values.

\begin{tabular}{|c|cc|ccc|}
\hline$\eta$ & LB & UB & MCP-g & $\begin{array}{c}\text { Construction } \\
\text { Phase }\end{array}$ & MCP_GRASP \\
\hline \hline 10 & 34651 & 34651 & 34589 & 34651 & 34651 \\
20 & 47068 & 48305 & 46780 & 46828 & 47133 \\
30 & 54083 & 55402 & 53921 & 54864 & 54988 \\
40 & 58472 & 60151 & 58284 & 58351 & 58550 \\
50 & 61817 & 63301 & 61676 & 61686 & 61817 \\
60 & 64167 & 65627 & 64081 & 64177 & 64157 \\
70 & 66147 & 67323 & 65992 & 66019 & 66147 \\
80 & 67510 & 68654 & 67473 & 67385 & 67625 \\
90 & 68802 & 69658 & 68594 & 68668 & 68802 \\
100 & 69770 & 70487 & 69538 & 69457 & 69756 \\
\hline
\end{tabular}

Table I

ABsolute VALUES - RESUlTS

Table I presents the absolute results, i.e., the number of achieved vehicles found by each approach. Field $\eta$ presents the number of RSUs that can be installed. The $L B C P L E X$ and the $U B C P L E X$ fields present the lower and upper bounds of the number of achieved vehicles found by CPLEX after 8 hours meaning that the optimal solution must be between these two values. Field $M C P$ - $g$ presents the solution - number of achieved vehicles - given by MCP-g algorithm. Field Construction Phase presents the number of achieved vehicles when the MCP_GRASP algorithm is run without the Local 
Search Phase. At last, the field MCP_GRASP presents the best MCP_GRASP solution.

\begin{tabular}{|c|c|c|c|c|}
\hline$\eta$ & $\begin{array}{c}\text { MCP_GRASP } \\
\text { Absolute } \\
\text { Gain (\%) }\end{array}$ & $\begin{array}{c}\text { Upper Bound } \\
\text { MCP_GRASP } \\
\text { Gap(\%) }\end{array}$ & $\begin{array}{c}\text { Upper Bound } \\
\text { MCP-g } \\
\text { Gap(\%) }\end{array}$ & $\begin{array}{c}\text { MCP_GRASP } \\
\text { Relative } \\
\text { Gap (\%) }\end{array}$ \\
\hline \hline 10 & 0.18 & 0.00 & 0.18 & 100 \\
20 & 0.75 & 2.43 & 3.16 & 23.90 \\
30 & 1.98 & 0.75 & 2.67 & 74.02 \\
40 & 0.46 & 2.66 & 3.10 & 14.70 \\
50 & 0.23 & 2.34 & 2.57 & 8.91 \\
60 & 0.27 & 2.09 & 2.36 & 11.66 \\
70 & 0.23 & 1.75 & 1.98 & 11.88 \\
80 & 0.23 & 1.50 & 1.72 & 13.10 \\
90 & 0.30 & 1.23 & 1.53 & 19.85 \\
100 & 0.31 & 1.04 & 1.35 & 23.29 \\
\hline
\end{tabular}

Table II

RELATIVE VALUES - RESULTS

Table II presents the relative results, i.e., the percentual bounds and gains. The field MCP_GRASP Absolute Gain $(\%)$ presents the percentual improvement when the MCP-g is compared to the MCP_GRASP algorithm. It is computed by the equation $\frac{M C P \_G R A S P-M C P-g}{M C P-g}$. The field Upper Bound MCP_GRASP Gap (\%) presents the percentual distance between the upper bound given by CPLEX and the MCP_GRASP solution. This field represents the maximal distance between the MCP_GRASP solution and the optimal solution, i.e., in the worst case the MCP_GRASP solution is Upper Bound MCP_GRASP Gap (\%) of the optimal solution. It is computed by the equation $\frac{U B C P L E X-M C P \_G R A S P}{U B C P L E X}$. The field Upper Bound MCP-g Gap (\%) presents the percentual distance between the upper bound given by CPLEX and the MCP-g solution. This field represents the maximal distance between MCP-g solution and the optimal solution, i.e., in the worst case the MCP-g solution is Upper Bound MCP-g Gap (\%) of the optimal solution. It is computed by the equation $\frac{U B C P L E X-M C P-g}{U B C P L E X}$.

In order to get a better representation for the effective gain of the MCP_GRASP algorithm, the MCP_GRASP Relative Gain (\%) is given. For instance, when $\eta=100$ the MCP_GRASP solution improves the MCP-g solution in only $0.31 \%$. This result does not seem relevant. However, when the Upper Bound MCP-g Gap (\%) is analysed, it is possible to see that the MCP-g solution is at most $1.36 \%$ of the optimal solution, i.e., no algorithm can improve the MCP-g solution more than $1.36 \%$. If the Upper Bound MCP-g Gap (\%) is considered as $100 \%$ - maximal improvement - the $0.31 \%$ of the MCP_GRASP Absolute Gain (\%) are $22.97 \%$ of the Upper Bound MCP-g Gap (\%), i.e., of the maximal possible gain.

The Figures 3 and 4 present the behavior of the MCP_GRASP algorithm when $\eta=100$ and $\alpha=4$ over all 100 iterations and compare with the MCP-g solution and CPLEX lower and upper bounds. The Figure 3 presents all 100 solutions given by MCP_GRASP algorithm. The black line is the MCP_GRASP solution; the dashed line is the constant
MCP-g solution and the gray line is the best MCP_GRASP solution found until each iteration.

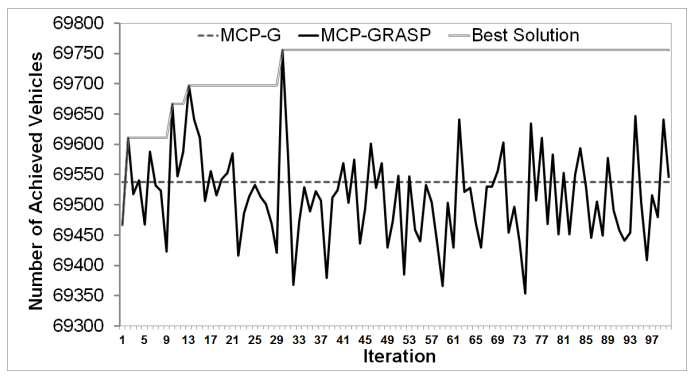

Figure 3. Number of Contacting Vehicles - MCP_GRASP Convergence

The Figure 4 presents the upper bound solution given by solver CPLEX, the lower bound given by MCP-g algorithm and the best solution over all 100 iterations given by MCP_GRASP algorithm.

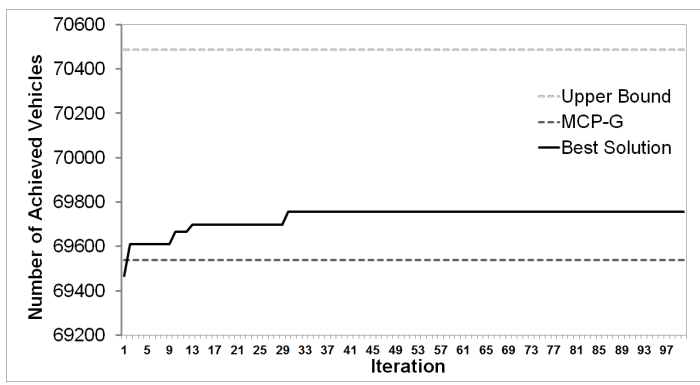

Figure 4. Number of Contacting Vehicles - Relative Gain

\section{B. Comparing Contact Distribution Over Time}

In this section, a new metric to measure the efficiency of a Deployment strategy is presented. This metric analyzes the contact distribution over time. It is considered that the more homogeneous the contact distribution over time, the better the quality of service. For instance, if with a specific Deployment 10000 vehicles contacts the RSUs over 60 minutes, it does not matter if the 10000 vehicles contacts RSUs is in the first 10 minutes and in the last 50 minutes there are no contacts. Of course, if all contacts occurs in a small period of time it can have data congestion impacting on the quality of service. On the other hand, if the contacts occurs in a more homogeneous way, the congestion can be minimized.

Although the MCP_GRASP algorithm does not explicitly take into account the contact distribution over time, this metric is analysed for 5 different Deployment solutions. In this analysis, it was simulated two hours traffic (from 6.30 am to $8.30 \mathrm{am}$ ) splitting the simulation in 120 equal parts (one for minute). At each minute the percentual of vehicles that are getting a contact opportunity over the total of vehicles that are running at the exact minute is compared.

The Figure 5 presents the percentual of connecting vehicles for $\eta$ equal to 20,40,60, 80 and 100. As can be seen, that Deployment strategy has a homogeneous behavior. Except at 
very early morning (15 initial minutes) when the percentual of connected vehicles is smaller, at each minute the percentual of vehicles contacting the infrastructure remains almost constant. Of course, when the number of RSUs is bigger, a better percentage of achieved vehicles can be reach.

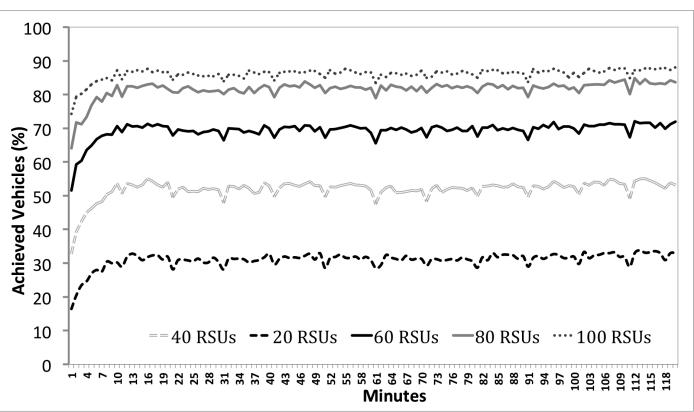

Figure 5. Percentage of Contacting Vehicles Over Time

\section{FINAL REMARKS}

This work deals with a facility location problem to vehicular networks. The goal is to find the best set of urban cells to install RSUs in order to maximize the number of distinct vehicles contacting the infrastructure in a VANET. Two different approaches to solve the problem is proposed. The first one is an ILP model that computes sharp lower and upper bounds even to instances with more than 75000 vehicles and 10000 possible urban cells. This model also found an optimal solution when $\eta=10$. The second one is a GRASP-based [23] heuristic, named MCP_GRASP which uses the MCP-g algorithm proposed by Trullols et al. [10] in its Construction Phase algorithm. Those two approaches are compared with the MCP-g algorithm.

An in-depth comparison among the three strategies considering the realistic vehicular mobility trace of the Cologne's city is performed. A new metric to measure the efficiency of a Deployment strategy is also proposed. This metric takes into account the percentual of vehicles that have a contact opportunity over the ones that are running in a period of time. The proposed MCP_GRASP algorithm seems to have homogeneous behavior over time.

Results demonstrate that: (i) the ILP can found sharp lower and upper bounds for the problem; (ii) the MCP-g algorithm [10] can found close-to-optimal solutions, a assumption not proved in the Trullols et al. [10] work; (iii) the proposed GRASP-like heuristic can found better solutions than the MCP-g algorithm and; (iv) the Deployment proposed by the GRASP-like heuristic has a homogenous behavior over time, i.e, the percentage of connected vehicles maintains almost constant over time.

As future works a genetic algorithm to solve this problem and new techniques to get optimal results to larger instances will be proposed.

\section{REFERENCES}

[1] H. Hartenstein and K. Laberteaux, "A tutorial survey on vehicular ad hoc networks," Communications Magazine, IEEE, vol. 46, pp. 164-171, June 2008.
[2] J. Barrachina, J. A. Sanguesa, M. Fogue, P. Garrido, F. J. Martinez, J.-C. Cano, C. T. Calafate, and P. Manzoni, "V2x-d: a vehicular density estimation system that combines $\mathrm{v} 2 \mathrm{v}$ and $\mathrm{v} 2 \mathrm{i}$ communications," in IEEE/IFIP Wireless Days, (Valencia, Spain), November 2013.

[3] G. Karagiannis, O. Altintas, E. Ekici, G. Heijenk, B. Jarupan, K. Lin, and T. Weil, "Vehicular networking: A survey and tutorial on requirements, architectures, challenges, standards and solutions," IEEE Communication Surveys \& Tutorials, vol. 13, no. 04, pp. 584-616, 2011.

[4] J. Blum, A. Eskandarian, and L. Hoffman, "Challenges of intervehicle ad hoc networks," Intelligent Transportation Systems, IEEE Transactions on, vol. 5, pp. 347-351, Dec 2004.

[5] A. Reis, S. Sargento, and O. Tonguz, "On the performance of sparse vehicular networks with road side units," in Vehicular Technology Conference (VTC Spring), 2011 IEEE 73rd, pp. 1-5, May 2011.

[6] K. Mershad, H. Artail, and M. Gerla, "Roamer: Roadside units as message routers in vanets," Ad Hoc Networks, vol. 10, no. 3, pp. $479-$ 496, 2012.

[7] Y. Wu, Y. Zhu, and B. Li, "Infrastructure-assisted routing in vehicular networks," in INFOCOM, 2012 Proceedings IEEE, pp. 1485-1493, IEEE, 2012

[8] A. Kchiche and F. Kamoun, "Centrality-based access-points deployment for vehicular networks," in Telecommunications (ICT), 2010 IEEE 17th International Conference on, pp. 700-706, IEEE, Doha 2010.

[9] J. Barrachina, P. Garrido, M. Fogue, F. J. Martinez, J.-C. Cano, C. T. Calafate, and P. Manzoni, "Road side unit deployment: A density-based approach," IEEE Intelligent Transportation Systems Magazine, vol. 5, pp. 30-39, Jul 2013.

[10] O. Trullols, M. Fiore, C. Casetti, C. Chiasserini, and J. B. Ordinas, "Planning roadside infrastructure for information dissemination in intelligent transportation systems," Computer Communications, vol. 33, no. 4, pp. $432-442,2010$.

[11] Y. Li, D. Jin, P. Hui, Z. Wang, and S. Chen, "Limits of predictability for large-scale urban vehicular mobility," Intelligent Transportation Systems, IEEE Transactions on, vol. 15, pp. 2671-2682, Dec 2014.

[12] D. Zhang, H. Huang, J. Zhou, F. Xia, and Z. Chen, "Detecting hot road mobility of vehicular ad hoc networks," Mobile Networks and Applications, vol. 18, no. 6, pp. 803-813, 2013.

[13] A. Sadiq, K. Bakar, K. Ghafoor, J. Lloret, and R. Khokhar, "An intelligent vertical handover scheme for audio and video streaming in heterogeneous vehicular networks," Mobile Networks and Applications, vol. 18, no. 6, pp. 879-895, 2013.

[14] O. Trullols-Cruces, M. Fiore, and J. Barcelo-Ordinas, "Cooperative download in vehicular environments," Mobile Computing, IEEE Transactions on, vol. 11, no. 4, pp. 663-678, 2012.

[15] Y. Liu, J. Niu, J. Ma, and W. Wang, "File downloading oriented roadside units deployment for vehicular networks," Journal of Systems Architecture, vol. 59, no. 10, Part B, pp. 938 - 946, 2013. Advanced Smart Vehicular Communication System and Applications.

[16] G. Marfia, G. Pau, E. De Sena, E. Giordano, and M. Gerla, "Evaluating vehicle network strategies for downtown portland: opportunistic infrastructure and the importance of realistic mobility models," in Proceedings of the 1st international MobiSys workshop on Mobile opportunistic networking, pp. 47-51, ACM, 2007.

[17] O. Tonguz and W. Viriyasitavat, "Cars as roadside units: a self-organizing network solution," Communications Magazine, IEEE, vol. 51, pp. 112-120, December 2013.

[18] H. Liang and W. Zhuang, "Cooperative data dissemination via roadside wlans," Communications Magazine, IEEE, vol. 50, pp. 68-74, April 2012.

[19] J. Lee and C. Kim, "A roadside unit placement scheme for vehicular telematics networks," in Advances in Computer Science and Information Technology (T.-h. Kim and H. Adeli, eds.), vol. 6059 of Lecture Notes in Computer Science, pp. 196-202, Springer Berlin Heidelberg, 2010.

[20] C. Jeonghee, J. Yeongwon, P. Hyunsun, H. Taehyeon, and P. Soyoung, "An effective rsu allocation strategy for maximizing vehicular network connectivity.," International Journal of Control \& Automation, vol. 6, no. 4, pp. $259-270,2013$.

[21] C. M. Silva, W. Meira, and J. F. M. Sarubbi, "Non-intrusive planning the roadside infrastructure for vehicular networks," IEEE Transactions on Intelligent Transportation System, vol. PP, pp. 1-10, Nov 2015.

[22] T. H. Cormen, C. E. Leiserson, R. L. Rivest, and C. Stein, Introduction to algorithms. MIT press, 2001.

[23] T. Feo and M. Resende, "Greedy randomized adaptive search procedures,” Journal of Global Optimization, vol. 6, pp. 109-135, 1995. 